\title{
Perinatal Outcome in Twin Pregnancies Complicated by Gestational Diabetes Mellitus: A Comparative Study
}

\begin{abstract}
The purpose of this study is to compare perinatal outcomes of twin pregnancies complicated by gestational diabetes (GDM) with those unaffected by GDM. A total of 1,154 twin pregnancies who delivered at Cheil General Hospital, between January 1998 and December 2002 were recruited to participate in a retrospective analysis. Out of these twin pregnancies, 37 women were had GDM. Four pregnancies exposed to GDM were excluded due to the loss of medical records; therefore 33 twin pregnancies exposed to GDM were enrolled. We matched the GDM pregnancies with pregnancies unaffected by GDM in a 1:2 ratio; therefore there were 33 GDM/66 without GDM who delivered during the study period. Our findings show that there were no significant differences including birth weight, Apgar score, respiratory distress syndrome, meconium aspiration pneumonia, transient tachypnea of new born, hyperbilirubinemia, hypoglycemia, hypocalcemia and congenital anomalies. Therefore, well controlled GDM may not increase perinatal complications in twin pregnancies. Careful pregnancy management and fetal surveillance in twin pregnancies is important to decrease perinatal complications and maintain a sound pregnancy and healthy offspring.
\end{abstract}

Key Words : Diabetes, Gestational; Pregnancy, Multiple; Perinatal Outcome; Pregnancy Outcome

\author{
Hye Jin Cho, Joong Sik Shin, \\ Jae Hyug Yang, Hyun Mee Ryu, \\ Moon Young Kim, Jung Yeol Han, \\ Joo Oh Kim, Hyun Kyong Ahn, \\ June Seek Choi, Jin Hoon Chung, \\ Su Hyun Park, Min Hyoung Kim, \\ Kyu Hong Choi
}

Department of Obstetrics and Gynecology, Cheil General Hospital and Women's Healthcare Center, Sungkyunkwan University School of Medicine, Seoul, Korea

Received : 16 May 2005

Accepted : 18 November 2005

Address for correspondence Joong Sik Shin, M.D.

Department of Obstetrics and Gynecology, Cheil General Hospital and Women's Healthcare Center, 1-19 Mukjeong-dong, Jung-gu, Seoul 100-380, Korea Tel : +82.2-2000-7127, Fax : +82.2-2000-7793 E-mail : jmjyjw@hanmail.net

\section{INTRODUCTION}

During the last two decades, the rate of multiple pregnancies has increased due to the expanded use of fertility stimulating therapy $(1,2)$. Increase in the frequency of multiple gestations is a concern because of the association with a variety of adverse pregnancy outcomes. The complication rates, of multiple gestations, correlates with the number of fetuses. The perinatal morbidity reported in twin gestations is two to three times higher than in singleton pregnancies $(3,4)$.

Gestational diabetes mellitus (GDM) is one of the most common medical complications observed during pregnancy (5) with an incidence of $2.2 \%$ in Korea (6). GDM has also been reported to be associated with adverse perinatal outcomes including macrosomia, neonatal hypoglycemia, jaundice, respiratory distress syndrome, polycythemia and hypocalcemia. All of these perinatal problems have been reported in neonates born to women with a history of $\operatorname{GDM}(6,7)$. GDM and twin pregnancies share several common risk factors such as, advanced maternal age, marked weight gain throughout gestation, and an increased frequency of maternal obesity. With respect to the etiology of GDM, several authors suggest pregnancy-related hormones such as gestagens, estrogens and human placental lactogen (hPL) which rise begin- ning in the 20th gestational week (8). In addition, the prevalence of gestational diabetes has been associated with increased placental mass and high hPL levels (8). In twin pregnancies, increased levels of these hormones may influence the frequency of GDM through their antagonistic actions on insulin (8). The increased risk of perinatal loss associated with twin pregnancies may be exacerbated by the additional adverse effects of superimposed GDM. However, there is limited information in the literature regarding this issue (9).

We hypothesized that altered carbohydrate metabolism would increase adverse perinatal outcomes in twin pregnancies in a manner similar to singleton pregnancies $(9,10)$. The purpose of this study was to compare perinatal outcomes between twin pregnancies complicated by GDM and those unaffected by GDM.

\section{MATERIALS AND METHODS}

A total of 1,154 twin pregnancies who delivered at Cheil General Hospital, between January 1998 and December 2002 were recruited to participate in a retrospective analysis. Out of a total of 1,154 twin pregnancies, 37 women had the diagnosis of GDM. Four twin pregnancies with GDM 
were excluded due to loss of the medical records; therefore there were 33 twin pregnancies affected by GDM that were enrolled in this study. Thirty three pregnancies with GDM were matched with 66 without GDM in a 1:2 ratio. All pregnancies delivered during the study period. The criteria used to match patients included chorionicity, maternal age, parity, body-mass-index (BMI) and gestational age at delivery.

Screening of all pregnant women between 24 and 28 weeks of gestation was carried out by measurement of serum or plasma glucose with the $50 \mathrm{~g}$ oral glucose challenge test (OGTT). A 1-hr post screen value of $130 \mathrm{mg} / \mathrm{dL}$ or more was considered positive and was followed by a $100 \mathrm{~g}$ diagnostic OGTT (11).

Diagnosis of GDM was based on the recommendations of the Fourth International Workshop-Conference on Gestational Diabetes, which adopts the Carpenter-Coustan criteria $(11,12)$. The plasma glucose threshold values were: 95 , 180, 155 and $140 \mathrm{mg} / \mathrm{dL}$ for fasting, 1-hr, 2-hr and 3-hr respectively. GDM was diagnosed whenever 2 or more of the above values were met or exceeded. All pregnant women diagnosed GDM were followed closely. Their care included individualized nutritional counseling from a qualified dietitian. This took into consideration a women's pre-pregnancy weight, activity level, dietary intake and weight gain, instructions on how to self-monitor glucose levels. All women were asked to check levels five times daily during pregnancy (fasting, two hours each postprandial and before bedtime). The recommended capillary blood glucose concentration for fasting or before bedtime levels were at least $60 \mathrm{mg} / \mathrm{dL}$ and no more than $95 \mathrm{mg} / \mathrm{dL}$ and levels two hours postprandial of no more than $120 \mathrm{mg} / \mathrm{dL}$. Insulin therapy was initiated if there were more than twenty percent exceeded the recommended capillary glucose levels despite diet therapy.

Maternal demographic characteristics, pregnancy risk factors and complications were examined in each group. Neonatal outcome variables including respiratory distress syndrome requiring mechanical ventilation, hyperbilirubinemia requiring transfusion or phototherapy and hypoglycemia (plasma glucose $<30 \mathrm{mg} / \mathrm{dL}$ ). Statistical analysis included the chi-

Table 1. Demographic comparisons between pregnancies with and without GDM

\begin{tabular}{lccc}
\hline & $\begin{array}{c}\text { Twin pregnancies } \\
\text { with GDM } \\
(\mathrm{n}=33)\end{array}$ & $\begin{array}{c}\text { without GDM } \\
(\mathrm{n}=66)\end{array}$ & $\begin{array}{c}p \\
\text { value }\end{array}$ \\
& $33.1 \pm 2.9$ & $32.9 \pm 3.1$ & $\mathrm{NS}$ \\
Maternal age (yr) & $0.2(0-2)$ & $0.2(0-1)$ & $\mathrm{NS}$ \\
Parity (median [range]) & $21.5 \pm 2.6$ & $21.0 \pm 2.2$ & $\mathrm{NS}$ \\
Prepregnant BMl (kg/m $)$ & $14.2 \pm 5.2$ & $16.3 \pm 5.1$ & $\mathrm{NS}$ \\
Weight gain during & & & \\
$\quad$ gestation (kg) & $36.2 \pm 2.1$ & $36.3 \pm 1.7$ & $\mathrm{NS}$ \\
\hline GA at delivery (weeks) & &
\end{tabular}

Values are presented as mean \pm standard deviation.

GDM, Gestational diabetes mellitus; BMI, Body mass index; NS, Not significant ( $p \geq .05)$; GA, Gestational age. square test and the Student t test. SPSS version 10.0 was used and a $p$-value below 0.05 was considered statistically significant.

\section{RESULTS}

During the study period, a total of 43,327 pregnant women delivered at our institution and 1,154 (2.7\%) were twin deliveries. Among these twin deliveries, 37 twin gestations were affected by GDM (3.2\%) and among 42,173 singleton deliveries, 921 pregnant women were affected by GDM (2.2\%). Demographic characteristics of the 33 twin pregnancies recruited to participate in this study are shown in Table 1 . There was no statistical difference in maternal age, parity, pre-pregnant BMI, weight gain during gestation and gestational age at delivery.

The comparison between perinatal complications in 33 cases of twin pregnancies complicated by GDM and 66 cases of twin pregnancies unaffected by GDM is shown Table 2 . No significant differences were found in regard to the birth weight, Apgar score, respiratory distress syndrome, meconium aspiration pneumonia, transient tachypnea of newborn, hyperbilirubinemia, hypoglycemia, hypocalcemia and congenital anomalies.

Twenty seven of the twin pregnancies affected by GDM were controlled without insulin therapy. Only 6 women needed insulin therapy to control GDM. All six patients treated with insulin had their blood glucose controlled within the recommended range.

Table 2. Peripartum complications of twin pregnancies with and without GDM

\begin{tabular}{lccc}
\hline & $\begin{array}{c}\text { Twins of mother } \\
\text { with GDM } \\
(\mathrm{n}=66)\end{array}$ & $\begin{array}{c}\text { Twins of mother } \\
\text { without GDM } \\
(\mathrm{n}=132)\end{array}$ & $\begin{array}{c}p \\
\text { value }\end{array}$ \\
\hline Mean birth weight (g) & $2440.3 \pm 530.3$ & $2423.9 \pm 397.7$ & $\mathrm{NS}$ \\
Apgar score $<7$ & & & \\
1 min & $3(4.8 \%)^{*}$ & $11(8.5 \%)^{\dagger}$ & $\mathrm{NS}$ \\
5 min & $0^{*}$ & $0^{\dagger}$ & $\mathrm{NS}$ \\
RDS & $15(22.7 \%)$ & $20(15.2 \%)$ & $\mathrm{NS}$ \\
MAP & $1(1.5 \%)$ & $1(0.8 \%)$ & $\mathrm{NS}$ \\
TTN & $2(3.0 \%)$ & $7(5.3 \%)$ & $\mathrm{NS}$ \\
Hyperbilirubinemia & $12(18.2 \%)$ & $29(22.0 \%)$ & $\mathrm{NS}$ \\
Hypoglycemia & $1(1.5 \%)$ & $1(0.8 \%)$ & $\mathrm{NS}$ \\
Hypocalcemia & $14(21.2 \%)$ & $20(15.2 \%)$ & $\mathrm{NS}$ \\
Congenital anomaly & $3(4.5 \%)$ & $3(2.3 \%)$ & $\mathrm{NS}$ \\
IUFD & $3(4.5 \%)$ & $2(1.5 \%)$ & $\mathrm{NS}$ \\
50 g-screening (mg/dL) & $163.3 \pm 20.6$ & $113.4 \pm 12.2$ & 0.000 \\
\hline
\end{tabular}

Values are presented as mean \pm standard deviation

GDM, Gestational diabetes mellitus; NS, Not significant ( $p \geq .05$ ); RDS, Respiratory distress syndrome; MAP, Meconium aspiration pneumonia; TTN, Transient tachypnea of newborn; NICU, Neonatal intensive care unit; IUFD, Intrauterine fetal death.

${ }^{*}$ The values excluded three infants of intrauterine fetal death. ${ }^{\dagger}$ The values excluded two infants of intrauterine fetal death. 
There were three cases of intrauterine fetal death in two diabetic patients both fetal deaths were in pregnancies with a monochorionic diamniotic twin gestation and a single fetal death was found in a dichorionic diamniotic twin gestation. In non-diabetic patients, there were two cases of single intrauterine fetal death, one in a monochorionic diamniotic twin gestation and the other in a dichorionic diamniotic twin pregnancy.

\section{DISCUSSION}

Clinical interest in the relationship between placental size and the incidence of GDM has stimulated numerous reports concerning the frequency of glucose intolerance in twin gestations. Although human placental lactogen levels have been found to be higher in twin pregnancies, several investigators using intravenous glucose tolerance test data concluded that twin gestation is not a risk factor for $\operatorname{GDM}(8,13-15)$. In this study, the incidence of GDM in twin pregnancies (3.2\%) was not significantly higher than that in singleton pregnancies $(2.2 \%)$. This observation is consistent with other reports (15).

It is well documented that twin pregnancy and GDM increase perinatal complications when both are present in a pregnancy $(5,16)$. Moreover, women who develop gestational diabetes have a 17 to 63 percent risk of developing overt diabetes mellitus within 5 to $16 \mathrm{yr}$ after the index pregnancy. Women who had marked hyperglycemia during or soon after pregnancy, who are obese, or whose GDM was diagnosed before 24 weeks of gestation have a particularly higher risk of developing overt diabetes (5). Twin pregnancies have a higher general perinatal risk; this may be exacerbated by GDM.

The purpose of this study was to compare perinatal outcomes between twin pregnancies complicated by GDM and those unaffected by GDM. However, GDM twin pregnancies did not show higher peripartum complications. A possible explanation for this finding might be that in our high risk clinics, there is a careful management protocol for GDM and multiple gestations. This most likely results in a lower rate of adverse perinatal outcomes.

Nevertheless, a much larger prospective study is needed to determine whether GDM twin pregnancies have increased perinatal complications despite good diabetic control. It is suggested that well controlled GDM may prevent perinatal complications in twin pregnancy. Therefore, intensive pregnancy management and fetal surveillance in twin pregnancies is important to decrease perinatal complications and maintain a sound pregnancy and healthy offspring.

\section{REFERENCES}

1. Jewell SE, Yip R. Increasing trends in plural births in the United States. Obstet Gynecol 1995; 85: 229-32.

2. Kiely JL, Kleinman JC, Kiely M. Triplets and higher-order multiple births, time trends and infant mortality. Am J Dis Child 1992; 146: 862-8.

3. Goldman M, Kitzmiller JL, Abrams B, Cowan RM, Laros RK Jr. Obstetric complications with GDM. Effects of maternal weight. Diabetes 1991; 40 (Suppl 2): 79-82.

4. Zhang J, Bowes WA Jr, Grey TW, McMahon MJ. Twin delivery and neonatal and infant mortality: a population-based study. Obstet Gynecol 1996; 88 (4 Pt 1): 593-8.

5. Kjos SL, Buchanan TA. Gestational diabetes mellitus. $N$ Engl Med 1999; 34: 1749-56.

6. Jang HC, Cho NH, Min YK, Han IK, Jung KB, Metzger BE. Increased macrosomia and perinatal morbidity independent of maternal obesity and advanced age in Korean women with GDM. Diabetes Care 1997; 20: 1582-8.

7. Persson B, Hanson U. Neonatal morbidities in gestational diabetes mellitus. Diabetes Care 1998; 21: B79-84.

8. Spellacy WN, Buhi WC, Birk SA. Human placental lactogen levels in multiple pregnancies. Obstet Gynecol 1978; 52: 210-2.

9. Keller JD, Utter GO, Dooley SL, Minogue JP, Keith LG. Northwestern University Twin Study X: Outcome of twin gestations complicated by gestational diabetes mellitus. Acta Genet Med Gemellol 1991; 40: 153-7.

10. Naidoo L, Jailal I, Moodley J, Desai R. Intravenous glucose tolerance tests in women with twin pregnancy. Obstet Gynecol 1985; 66: 500-2.

11. Buhling KJ, Henrich W, Starr E, Lubke M, Bertram S, Siebert G, Dudenhausen JW. Risk for gestational diabetes and hypertension for women with twin pregnancy compared to singleton pregnancy. Arch Gynecol Obstet 2003; 269: 33-6.

12. Metzger BE, Coustan DR. Organizing Committee. Summary and recommendations of the Fourth International Workshop-Conference on Gestational Diabetes. Diabetes Care 1998; 21: B161-7.

13. Spellacy WN, Handler A, Ferre CD. A case-control study of 1253 pregnancies from a 1982-1987 perinatal data base. Obstet Gynecol 1990; 75: 168-71.

14. Wein P, Warick MM, Beischer NA. Gestational diabetes in twin pregnancy: prevalence and long-term implications. Aust NZ J Obstet Gynecol 1992; 32: 325-7.

15. Henderson CE, Scarpelli S, LaRosa D, Divon MY. Assessing the risk of gestational diabetes in twin gestation. J Natl Med Assoc 1995; 87: 757-8.

16. Dickinson JE, Palmar SM. Gestational diabetes: Pathophysiology and diagnosis. Sem Perinatol 1990; 14: 2-11. 